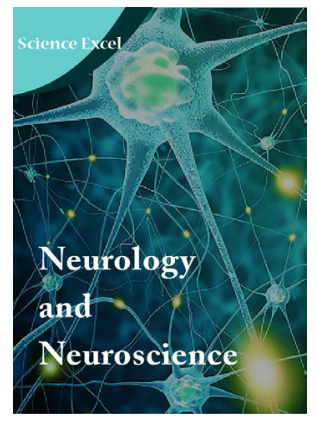

Correspondence

Prof. Ivet KOLEVA, MD, PhD, DMedSc Str. «Dospat» 6 ; Sofia 1606, Bulgaria Tel: +359.888.20 8161

E-mail:yvette@cc.bas.bg; dr.yvette.5@gmail. com

\footnotetext{
- Received Date: 09 April 2020;

- Accepted Date: 13 April 2020

- Publication Date: 18 April 2020.
}

\section{Keywords:}

Cervical Spine injury; Physical and Rehabilitation medicine; Physiotherapy; Stabilometry; Whiplash associated disorder

\section{Abbreviations}

BMI: Body-Mass Index; BPI: Biomechanical postural index; COF: Center of Pression Angle; COP: Center of Pression; ICF: International Classification of Functioning; LAB: Laboratory results; MF: Magnetic field; MMT: Manual Muscle Test; OT: Occupational therapy; PRM: Physical and Rehabilitation Medicine; PT: Physiotherapy; QTF: Quebec Task Force for WAD; ROM: Range of Motion; TENS: Transcutaneous Electro-NeuroStimulation; VAS: Visual Analogue Scale; WAD: Whiplash associated disorders

\section{Copyright}

(c) 2020 Science Excel. This is an openaccess article distributed under the term of the Creative Commons Attribution 4.0 International license.

\title{
Stabilometry and balance training for functional recovery and rehabilitation of a patient with a whiplash associated disorder
}

\author{
Koleva Ivet ${ }^{1,2^{*}}$, Hadjiyanev Assen ${ }^{3}$, Yanev Stefan ${ }^{1}$ and Yoshinov Borislav ${ }^{4}$ \\ ${ }^{\prime}$ Medical University of Sofia, Physiotherapy Department, Sofia, Bulgaria \\ ${ }^{2}$ Multi-profile Hospital for Long-term care and rehabilitation "Serdika", Department of Physical and Rehabilitation Medicine, \\ Sofia, Bulgaria \\ ${ }^{3}$ Multi-profile University Hospital "St Ivan Rilski", Neurosurgery Clinic, Sofia, Bulgaria \\ ${ }^{4}$ Sofia University, Medical Faulty, Sofia, Bulgaria
}

\begin{abstract}
By definition, Whiplash is a bony or soft tissue injury, resulting from rear-end or side impact, predominantly in motor vehicle accidents, consequence of "an acceleration-deceleration mechanism of energy transfer to the neck". The incidence of Whiplash associated disorders (WAD) increase during last years. Sometimes, clinical practice reveals rare signs, symptoms or complications of this clinical pattern.

We present a female patient of 25 years, transferred t our PRM Department one week after traffic accident, with persistent clinical manifestations, including excessive pain and stiffness in the neck region and muscles around it; headache, arm tingling; balance and gait instability; attention, concentration and memory troubles; reactive depression and anxiety. During clinical exam (at the admission) we diagnosed: vertebral and cervico-brachial radicular syndromes, balance and gait instability, positive Romberg. Spine X-Ray demonstrates osteochondrosis and spondylosis, static disturbances with reduction of the physiological cervical lordosis. Magnetic Resonance Imagery (MRI) of the cervical spine revealed multi-level discal herniation (predominantly C5-6 and C6-7). During Computerized Stabilometry, we observed altered center-of-Pressure (COP) trajectories and COP-oscillations with open and closed eyes.

We applied a complex rehabilitation, including paravertebral infiltrations, preformed physical modalities (TENS and Magnetic field), individualized physiotherapeutic and occupational therapeutic programme, balance and gait training, patient education.

We noticed significant efficacy of the rehabilitation: improvement of the range of motion of the cervical spine, pain relief, balance and gait stabilization, amelioration of autonomy in activities of daily life.

Our opinion is, that every patient after traffic accident needs consultation with a medical doctor - specialist in Neurology and in Physical and rehabilitation medicine. We consider that early rehabilitation must be considered in cases with whiplash-associated disorder. We emphasize on the impact of stabilometry for objective evaluation of the equilibrium disturbances. Some recommendations for the rehabilitation complex are formulated.
\end{abstract}

\section{Introduction}

By definition, Whiplash is a "bony or soft tissue injury", resulting from "rear-end or side impact, predominantly in motor vehicle accidents", consequence of "an acceleration-deceleration mechanism of energy transfer to the neck" [1-3]. Clinical signs and symptoms are included in the clinical pattern "Whiplash associated disorders (WAD)": headache, neck pain and stiffness, arm pain and paresthesia, memory and concentration troubles, reactive depression and anxiety. The Quebec Task Force (QTF) on WAD classified the severity of clinical manifestations in five grades: from 0 (no physical signs) to 4 (neck complain and fracture or dislocation) [3].

The incidence of WAD in developed countries have increased during recent decades, especially in case of low speed motor vehicle crashes [4]. The consequence is an increase of the patients' number and the appearance of atypical signs, symptoms or complications; some of them - unavoidable despite emergency staff's attention.

In these cases (with neurological signs and symptoms), the necessity of consultations with other medical specialists (in Neurology, Neurosurgery, Physical and Rehabilitation medicine /PRM/) is imposed.

Routinely, the treatment of patients with WAD includes soft collars and rest, methylprednisolone or local anesthetic facet blocks, in some cases percutaneous radiofrequency neurotomy $[5,6]$. Specific exercises strategies have not be studied [5].

We consider that special attention must be dedicated to patients with persistent and atypical clinical patterns; some of them - transferred from acute clinic to rehabilitation department at an ever-earlier stage (three days to one week after the car accident).

Citation: Ivet $\mathrm{K}$, Assen $\mathrm{H}$, Stefan $\mathrm{Y}$, et al. Stabilometry and balance training for functional recovery and rehabilitation of a patient with a whiplash associated disorder. Neurol Neurosci. 2020;1 (2):1-5 
In all patients, we realize detailed functional assessment and specific rehabilitation programme. The principal goal of every rehabilitation programme is: amelioration of the patient's state and his functional recovery; grasp / grip and gait training; resocialization of the patient and restoration of his / her autonomy in everyday life [7]. In posttraumatic rehabilitation, major tasks are oriented to: pain management, improvement of the joints' range of motion (ROM - active and passive), gait recovery with control of weight bearing and with technical aids [810]. In neurorehabilitation, the accent is stroked on muscle deficiency; on transfers, balance and gait stability $[9,10]$.

The appearance of atypical signs or unexpected complications can provoke an important delay or even suspension of the normal rehabilitation process. This is the present case.

The principal objective of the current article is to remind the possible occurrence of some rare symptoms, as postural instability and balance disturbances, in a patient after whiplash traumatic injury.

\section{Case Presentation}

\section{Patient's presentation}

The presented patient is a 25 years old female. Transferred to our PRM Department one week after the acute accident, with the objective of complex rehabilitation; for persistent clinical manifestations.

\section{Patient complaints at the moment of admission}

Patient suffers from excessive pain and stiffness in the neck region and muscles around it; arm tingling; headache; balance and gait instability; and subsequent altered autonomy in everyday life.

No active co-morbidities. No previous diseases.

No obesity. BMI = 23,4.

No previous history of risk factors.

The mother of the patient has hypertension and diabetes type 2 (developed tardily in her life). No family history of other risk factors for cerebro-vascular conditions.

Not at all addictions to alcohol or tobacco use.

\section{Clinical exam before rehabilitation}

Vertebral syndrome in the cervical region (with reduced cervical lordosis, paravertebral muscle spasm, limited range of motion of the cervical spine);

Bilateral cervico-brachial radicular syndrome (C5-8) with positive sensory signs (occipital headache, palpatory pain in the points of Valleix - in the cervical region and in upper extremities, paresthesia in C6 and C8 dermatomes); No reflex alterations, No muscle weakness;

Bilateral lumbo-sacral radicular syndrome (L5-S1) with positive and negative sensory signs (pain in the points of Valleix - in the lumbar region and in lower extremities, positive sign of Lassegue bilaterally at 75 degrees; hypoesthesia in L5 and S1 dermatomes); Reduction of the Aquilles reflex. No muscle weakness.

Otoneurological syndrome with equilibrium and gait instability, positive Romberg.

Psychological disturbances active attention troubles, concentration difficulties, memory alterations; reactive depression and anxiety (due to balance instability).

\section{Functional assessment before rehabilitation}

Limited range of motion (ROM) of the cervical spine: active flexion - $75^{\circ}$, passive flexion - $90^{\circ}$ (with tolerable pain); active extension was reduced to $10^{\circ}$; latero-flexion $-30 \mathrm{o}$; left and right rotations are limited to $45 \mathrm{o}$.

Manual muscle test (MMT) for cervical paravertebral muscles and muscles of upper extremities: $\mathrm{MMT}=3+/ 5$ for $\mathrm{m}$.supraspinatus utriusque (utr. - bilaterally) \& MMT 4/5 for sterno-cleido-mastoideus utr.

Reduced capacity for autonomic gait: possible only with assistance, due to altered equilibrium.
The pain was evaluated using Visual Analogue Scale (VAS) - from 0 ${ }^{*}$ no pain ${ }^{*}$ to $10{ }^{*}$ intensive pain". Before PRM, the patient suffered from intensive pain in different body positions: lying, sitting and standing straight (in bed - VAS $=5 / 10$; in vertical position $-\mathrm{VAS}=7 / 10$ ), and during neck movements (VAS=9/10).

\section{Assessment according the International Classification of Functioning, Disability and Health (ICF) [11]}

- Impairments of body functions and changes in body structures: headache, cervical paravertebral pain, reduced spine mobility, muscle weakness, and restricted ROM; attention and memory troubles;

- Activity limitation - altered balance stability and limited walking ability;

- Participation restrictions - reduced participation in leisure activities;

- Reduction of the patient's level of autonomy.

Biological constants: RR 120 / 85 mm Hg, frequency - 64 beats/min., saturation $-100 \%$.

LAB exam: normal Hb, Leuco, Thrombo; CRP, ionogram, lipid and glucidic patterns.

$X$-Ray imagery of the spine: Cervical and lumbar osteochondrosis and spondylosis. Static disturbances with significant alteration of the physiological cervical lordosis.

Magnetic Resonance Imagery (MRI) of the cervical spine: Cervical osteochondrosis and spondylosis. Multi-level discal herniation, predominantly at levels C5-6 and C6-7.

Figures 1, 2 and 3 present images of the patient's cervical spine (X-ray radiography and magnetic resonance imagery - MRI) after the whiplash traumatic event.
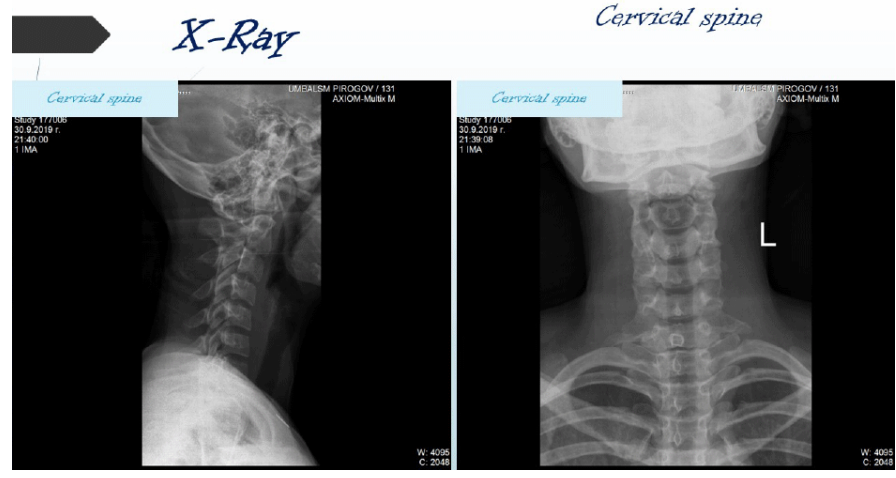

Figure 1. Radiographies of the patient's cervical spine - sagittal (a) and frontal (b) plane (static disturbances with important decrease of the cervical lordosis)

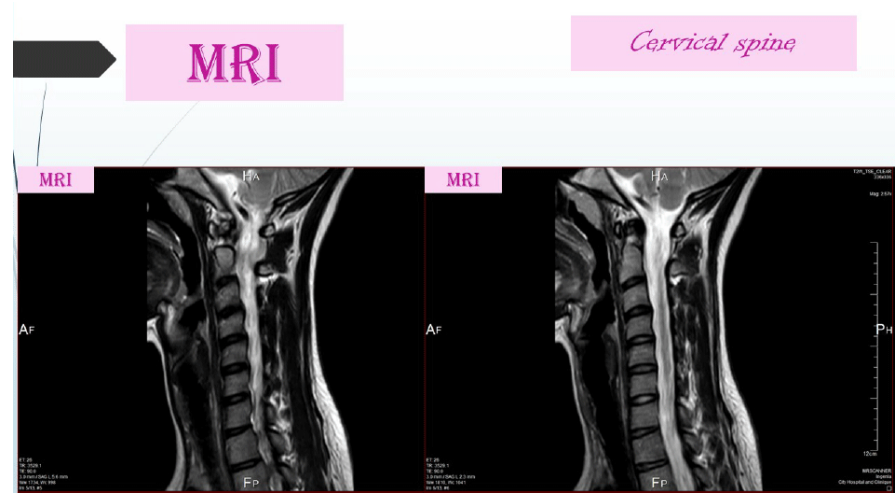

Figure 2. MRI of the patient's cervical spine (no cervical lordosis, multi-level discal herniation) 


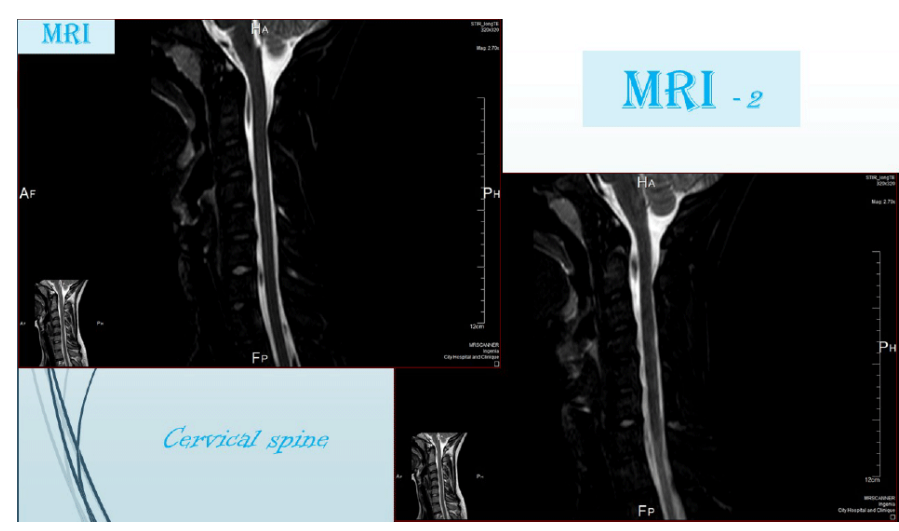

Figure 3. MRI of the cervical spine (T2)

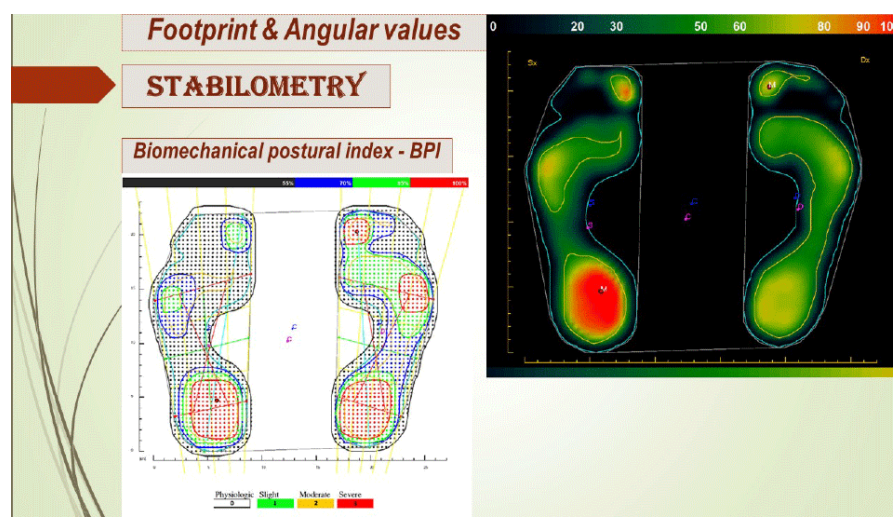

Figure 4. Stabilometry : $a /$ Footprint and b/ Biomechanical postural index

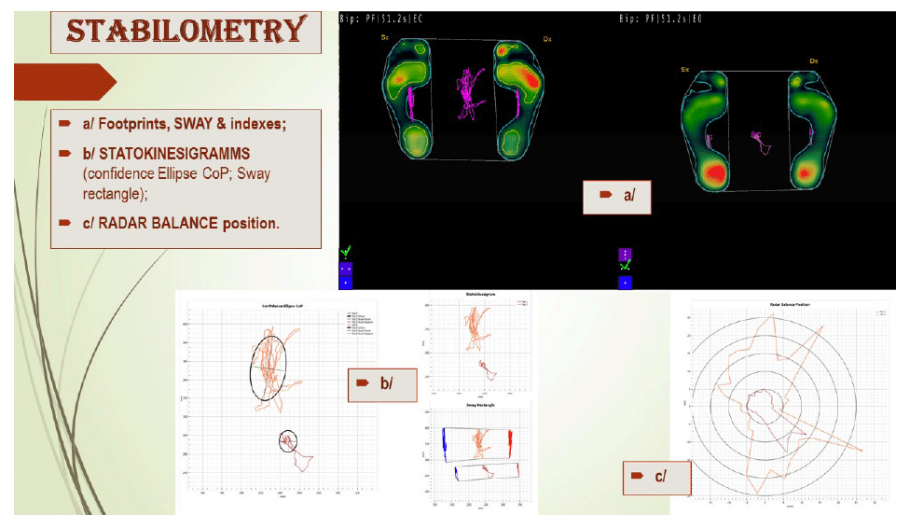

Figure 5. Stabilometry: sway [a], statokinesiograms [b] and radar balance position [c]

For objectification of balance instability we realized Clinical stabilometry analysis (Figures 4 and 5), executed according standard protocols [12,13]. We observed altered Center-of-Pressure (COP) trajectories and COP-oscillations with open and closed eyes.

Figure 4-a (right) presents the footprint and angular values: Center of pressure angle (COF), plantar angles (Long /LPA/ and Transversal / TPA/), Maximum pressure, Mean pressure, Pressure surfaces (forefoot, midfoot, rear foot). Figure 4-b (left) presents Biomechanical postural index (BPI) and isobaric foot map: Center of Pressure (COP) geometric position, Bipodal Load symmetry, COF angles, foot axis angle.

Figure 5-a (right) presents footprint, sway and respective indexes; figure 5-c - the radar balance position. In figure 5-b we can observe and analyze the difference in the oscillations of the center of pressure with closed and open eyes: confidence Ellipse-CoP and Sway rectangle.
PRM Program of Care $[9,10,14,15]$

\section{Goal}

The complex NR program accentuates on posture, balance and gait training.

Tasks

- $\quad$ recovery of the stability / equilibrium stabilization;

- retrieval of spine flexibility and mobility;

- restoration of the muscle and ligament balance, accentuating on muscles around the cervical spine;

- control of pain, ROM, muscle force;

- prevention of possible complications;

- autonomic gait recovery with amelioration of autonomy in everyday life;

- activities of daily living ( $A D L)$ training;

- $\quad$ psycho-emotional stimulation;

- enhancement of the health-related quality of life;

- resocialization.

Methods

- Per oral antalgics - Paracetamol or Paratramol, per os;

- Paravertebral infiltrations - with Cortisone, Lidocaine and $B$-vitamins (B1, B6, B12) - 5 applications;

- Patient's education;

- Posture (activity modification),

- Preformed physical modalities: Transcutaneous electro-neurostimulation (TENS) and low intensity low frequency Magnetic field (MF) for pain relief;

- Massage - classic relaxing paravertebral massage for the cervical region;

- Individualized physio-therapeutic (PT) programme: correct spine position, cervical spine tractions and upper limb joint mobilization (active range of motion), analytic exercises for muscles flexors and rotators of the cervical spine (accentuating on sternocleido-mastoideus muscles); post-isometric relaxation /PIR/ for upper trapezius muscle;

- Balance and gait training without technical aids; education in mobility with obstacles, up and down the stairs.

- Occupational therapy (OT) \& ADL training.

Results of the Applied PRM Programme and Future Recommendations after the Rehabilitation

We Observed:

- Amelioration of the ROM of the cervical spine: active flexion 90o, left and right latero-flexion - 60o; rotations - at 75o;

- $\quad$ Pain relief in cervical spine and upper extremities - VAS 2/10;

- Amelioration of the functional capacity: 10 meters walk test-5,3 seconds;

- Independent gait with collar but without technical aids or assistance;

- Balance \& Gait stabilization;

- Amelioration of the autonomy in ADL.

\section{Recommendations after the Rehabilitation course}

The treatment plan after the departure from the hospital includes:

- $\quad$ Auto-PT at home: physiotherapy every day at the 3-th month after the injury; Analytic exercises for the paravertebral muscles (muscle belt) and for muscles of upper extremities; Balance training;

- Next rehabilitation course - 3 months later. 


\section{Discussion}

\section{The current case}

The number of WAD in developed countries is growing and the increase of the patients' number is inevitable. The teamwork in these cases is obligatory. The early rehabilitation is needed.

The observed balance instability is a relatively rare, but logical and dangerous complication in patients after car accident with whiplash disorder. We must begin to think about this possibility in all patients after traffic accidents.

\section{Posture, Equilibrium and Stabilometry}

The posture is the position of a person's body or body parts. The term derives from the classical Latin noun "positura", meaning pose or position [16].

The term Equilibrium contains a root from the Latin Libra, meaning "weight" or "balance", and in all sciences, the special meaning refers to the balance of competing influences or forces [16].

The ability of the human body to retain the stable position or Balance is clinically assessed by the test of Romberg (swaying of the body when the feet are placed close together and the eyes are closed).

Routinely, qualitative assessment is sufficient for the clinical practice. However, in case of mild disorders or latent alterations a quantitative evaluation is preferred.

The study of the postural equilibrium is called Static posturography; Stabilography or Computerized stabilometry. According definitions, stabilometry represents the objective assessment of body sway during quiet standing - stance in the absence of any voluntary movements or external perturbations [17]. Practically, this is the objective analysis of the functioning of the postural control system $[18,19]$. The method is very easy to perform and its efficacy is significant.

\section{The PRM complex}

According to the definition of the European Union of Medical Specialists - PRM Section [8,14] Physical and Rehabilitation Medicine (PRM) is an independent medical specialty, oriented to the promotion of physical and cognitive functioning, activities (including environment), participation (including quality of life) and changes in personal factors and environment. The specialty PRM is responsible for the management of the prevention, diagnostics, treatment and rehabilitation of patients with health-related disability and co-morbidity of all ages.

According to the White Book on Physical and Rehabilitation Medicine [1] the basic objective of PRM is the optimization of social participation and the amelioration of the quality of life of patients. This includes the aid of the patient to reach possible levels and patterns of autonomy and independence, including participation in professional, social and leisure activities, part of his human rights $[8,9]$.

Tasks of PRM are: treatment of existing pathology; reduction of disability; prevention and therapy of complications; amelioration of functioning and activity; stimulation of patient's participation in different types of activities $[8,9,14]$.

In neurologic conditions (including after traumatism), during clinical assessment we emphasize on some specific analyses:

- $\quad$ pain (localization, type, intensity - verbal or visual analogue scale; modifying pain activities);

- $\quad$ evaluation of the muscle force / muscle weakness, motor deficit dynamometry, manual muscle test, weakness scales;

- fatigue (physical endurance, necessity of rest during the examination or the functional activity);

- coordination investigation (assessment of static, locomotory and dynamic ataxia);
- joint stability (including joint position sense) and range of motion (active and passive); presence of oedema, muscle or joint contractures;

- $\quad$ analysis of the grasp, balance and gait;

- mobility (necessity of technical aids - canes, walking sticks, crutches, walkers, wheelchairs and other devices);

- autonomy in everyday activities (bathing, dressing, eating, putting shoes on, personal hygiene, necessity of help in everyday activities). Evaluation of problems must be qualitative and quantitative, including: fatigue, motor deficiency, coordination problems (body posture, balance, gait, grasp); pain; conscience for the necessity of technical aids; difficulties in ADL; limitations in functional mobility $[9,10]$.

The complexity of neurorehabilitation imposes the necessity of a holistic approach to the patient - detailed functional analysis before and after the rehabilitation courses; application of therapeutic methods of different medical specialties (principally neurology and neurosurgery; orthopedics and traumatology; rheumatology; PRM) and from non-medical fields (physiotherapy, occupational therapy, sociology, psychology). We apply basic principles of the specialty Physical and Rehabilitation medicine $[8,15]$.

In every phase of the recovery process, we must define precisely the goal, tasks and algorithms of rehabilitation. In every case, our goal is to assure a high quality of the rehabilitation, optimal for the clinical form of the principal disease or condition, adapted to the age, co-morbidities, capacity and preferences of the concrete patient; with the strategic objective to receive the best result for his quality of life. We must put emphasis on balance and gait training, autonomy in everyday activities, pain control $[9,10,15]$.

\section{Conclusion}

Current case demonstrate that in every patient after cervical traumatic event we must consider a neurological consultation and an early rehabilitation.

\section{Ethical issues}

All authors declare that the work have been approved by the appropriate ethics committee and have therefore been performed in accordance with the ethical standards of the 1964 Declaration of Helsinki. All authors hereby declare that 'written informed consent' was obtained from the patient for publication of this case report and accompanying images.' Protection of privacy of the patient is guaranteed.

\section{Conflicts of interest}

Authors have declared that no competing interests exist.

\section{Acknowledgments}

No funding sources. No financial disclosures.

\section{References}

1. Barnsley L, Lord SM, Bogduk N. Whiplash injury. Pain 1994;58:283-307.

2. Yadla S, Ratliff JK, Harrop JS. Whiplash: diagnosis, treatment, and associated injuries. Curr Rev Musculoskelet Med 2008;1:65-68.

3. Spitzer WO, Skovron ML, Salmi LR, et al. Scientific monograph of the Quebec task force on whiplash-associated disorders: redefining "Whiplash" and its management. Spine 1995;20 (8S):1S-73S.

4. Uhrenholt L, Gregersen M. Low-speed Motor Vehicle Crashes--Marginal Values for Whiplash-Associated Disorders. Ugeskr Laeger, 2008;170(9):713-715

5. Rodriguez AA, Barr KP, Burns SP. Whiplash: Pathophysiology, Diagnosis, Treatment, and Prognosis. Muscle Nerve 2004;29(6):768-81.

6. Vendrig AA, van Akkerveeken PF, McWhorter KR. Results of a multimodal treatment program for patients with chronic symptoms after a whiplash injury of the neck. Spine 2000;25:238-44.

7. World Health Organization and the World Bank. World Report on Disability. Geneva: WHO Press, 2011.

8. White Book on Physical and Rehabilitation Medicine in Europe. Produced by the Section of Physical and Rehabilitation Medicine, Union Européenne des Médecins 
Spécialistes (UEMS), the European Board of Physical and Rehabilitation Medicine and l'Académie Européenne de Medicine de Réadaptation in conjunction with the European Society of Physical and Rehabilitation Medicine (ESPRM). C Gutenbrunner, AB Ward, MA Chamberlain Editors. Journal of Rehabilitation Medicine 2007;1 (Supple 45):1-48.

9. Koleva I. Repetitorium physiotherapeuticum (basic principles of the modern physical and rehabilitation medicine). Book for English speaking students of Pleven Medical University. - Sofia: Publishing house "SIMEL". 2006; 95 p.

10. Grasp and Gait rehabilitation (Bases). Monograph. Edited by prof. Ivet Koleva and prof. Taina Avramescu. - Sofia:'SIMEL Press', 2017, 396 pages. Printed version: ISBN: 978-619-183-055-8.

11. World Health Organization. International Classification of Functioning, Disability and Health (ICF). - Geneva, WHO; 2001.

12. Scoppa F, Capra R, Gallamini M, Shiffer R. Clinical stabilometry standardization: Basic definitions - Acquisition interval - Sampling frequency. Gait \& Posture 2013; 37(2):290-292.

13. Collins JJ, De Luca CJ. Open-loop and Closed-Loop Control of Posture: A Random-
Walk Analysis of Center-Of-Pressure Trajectories. Exp Brain Res 1993;(95)2:308-318.

14. White Book on Physical and Rehabilitation Medicine in Europe. Third Edition. 2018. European PRM Bodies Alliance: Euroepan Academy of Rehabilitation Medicine, European Society of PRM, EUMS - PRM Section, European College of PRM. European Journal of Physical and Rehabilitation Medicine 2018;54(2):1-204

15. Koleva I, Yoshinov B, Yoshinov R. Perspectives in Pain Management: Physical Analgesia. Medical Journal of Clinical Trials \& Case Studies 2018;2(2):1-3.

16. The American Heritage ${ }^{\circledast}$ Dictionary of the English language. Fifth edition Houghton Mifflin Harcourt Publishing Company, 2016.

17. Chiari L. Stabilometry. - In: Binder M.D., Hirokawa N., Windhorst U. (Editors) Encyclopedia of Neuroscience. Berlin - Heidelberg, Springer, 2009, pp.28-324.

18. Kapteyn TS, Bles W, Njiokiktjien CJ, Kodde L, Massen CH, Mol JM. Standardization in platform stabilometry being a part of posturography. Agressologie 1983;24:321 326.

19. Winter DA. Human balance and posture control during standing and walking. Gait Posture 1995; 3:193-214. 